\title{
BMJ Open Use of corticosteroids during pregnancy and risk of asthma in offspring: a nationwide Danish cohort study
}

\author{
Anna Byrjalsen, Trine Frøslev, Ane Birgitte Telén Andersen, Morten Olsen, \\ Henrik Toft Sørensen
}

To cite: Byrjalsen A, Frøslev T, Telén Andersen AB, et al. Use of corticosteroids during pregnancy and risk of asthma in offspring: a nationwide Danish cohort study. BMJ Open 2014;4: e005053. doi:10.1136/ bmjopen-2014-005053

- Prepublication history and additional material is available. To view please visit the journal (http://dx.doi.org/ 10.1136/bmjopen-2014005053)

Received 13 February 2014 Revised 21 April 2014 Accepted 13 May 2014

\section{CrossMark}

Department of Clinical Epidemiology, Aarhus University, Aarhus N, Denmark

Correspondence to Dr Anna Byrjalsen; a.byrjalsen@hotmail.com

\section{ABSTRACT}

Objective: To examine whether in utero exposure to local and systemic corticosteroids is associated with asthma development in offspring.

Design: Cohort study.

Setting: Denmark.

Participants: We included all singletons born alive in Denmark between 1996 and 2009. Data on maternal corticosteroid use, asthma in offspring and covariates were obtained from medical registries.

Main outcome measures: We compared asthma risks of children prenatally exposed to corticosteroids and of children of former corticosteroid users with that of unexposed children. We computed absolute risks and used proportional-hazards regression to compute adjusted HRs (aHRs). Using logistic regression we compared exposed children with unexposed siblings in a 'within-mother-between-pregnancy' analysis. Adjustment addressed varying length of follow-up. Results: We identified 877778 children, $3.6 \%$ of whom were prenatally exposed to systemic $(n=5327)$ or local ( $n=24436)$ corticosteroids. A total of 105677 children developed asthma during follow-up with a 10 year risk of $18.4 \%$ among the exposed and $13.5 \%$ among the unexposed. The aHR was $1.54(95 \% \mathrm{Cl}$ 1.45 to 1.65$)$ for systemic use, $1.45(95 \% \mathrm{Cl} 1.40$ to 1.50) for local use and $1.32(95 \% \mathrm{Cl} 1.30$ to 1.34$)$ for former use. The adjusted OR of the 'within-motherbetween-pregnancy' analysis was $1.11(95 \% \mathrm{Cl} 0.98$ to 1.25).

Conclusions: These population-based data do not support a strong causal association between maternal corticosteroid use during pregnancy and increased asthma risk in offspring.

\section{INTRODUCTION}

Asthma has become the most common chronic lower respiratory tract disease of childhood, with an estimated prevalence of $5 \%$ to $20 \%$ among children aged 13-14 in industrialised countries. ${ }^{1}$ The causes of asthma are largely unknown; however, genetics and maternal factors such as smoking and high body

\section{Strengths and limitations of this study}

- The strengths of the study are the large population size, the long and complete follow-up and the use of data from medical databases with no risk of recall bias and minimal risk of selection bias.

- The limitations are misclassification of exposure status, the fact that prescription dispensations may be an imperfect measure of disease and unmeasured confounding.

mass index (BMI) have been reported as risk factors. $^{2-4}$ Furthermore, use of paracetamol, proton pump inhibitors (PPIs), cyclooxygenase (COX) inhibitors and antibiotics during pregnancy also has been associated with asthma in offspring. ${ }^{5-8}$ Proposed pathophysiological mechanisms underlying asthma development include alterations in the developing immune system ${ }^{9}$ which may be affected by maternal corticosteroid intake. ${ }^{10}$

Corticosteroids are potent anti-inflammatory drugs used during pregnancy for conditions such as asthma and inflammatory bowel disease (IBD). Reported fetal side effects include altered development of the hypothalamic-pituitary-adrenal axis and abnormal organ function. ${ }^{11-13}$ A Canadian study found that antenatal corticosteroid therapy used to induce fetal lung maturation was associated with childhood asthma (adjusted OR $(\mathrm{aOR})=1.23(95 \%$ CI 1.06 to 1.44$)) .{ }^{14}$

We included oral corticosteroids and injections (systemic use). Nasal sprays, eyedrops, eardrops, local treatment in the mouth, inhalation corticosteroids, intestinal foams and suppositories were included as local use. Creams, over-the-counter medication (nasal spray and creams) and in-hospital administered corticosteroids were excluded. 
Given the widespread use of corticosteroids it is of major public health and clinical importance to gain knowledge about the effects of corticosteroid therapy on the fetus during the entire pregnancy. To the best of our knowledge, no studies to date have investigated the association between maternal corticosteroid therapy at any time during pregnancy and asthma in offspring. We therefore conducted a cohort study based on nationwide Danish medical registries, to examine the association between maternal corticosteroid therapy during pregnancy and the postnatal period and risk of asthma in the offspring.

\section{METHODS}

\section{Setting and study population}

This nationwide cohort study included all singletons born alive in Denmark from 1 January 1996 until 31 December 2009. The children and their parents were identified through the Danish Medical Birth Registry (DMBR), which stores data on all births in Denmark since $1973 .{ }^{15}{ }^{16}$ We used the civil registration number (CRN), a unique 10-digit personal identifier issued to every Danish citizen at birth or upon immigration, ${ }^{15}$ to perform unambiguous linkage between registries. In Denmark, the National Health Service provides taxsupported healthcare to all residents and refunds a portion of patient expenditures for a wide range of prescribed drugs, including corticosteroids.

\section{Corticosteroid use}

Exposure to corticosteroids during pregnancy was defined as redemption of at least one maternal prescription for a systemic corticosteroid or at least two prescriptions for local corticosteroids 30 days before or during pregnancy. We established the start of pregnancies based on data on gestational age and birth date from the DMBR.

We further categorised prenatal exposure into exposure during the first trimester (the first 12 weeks of pregnancy) and during the remainder of pregnancy. Children exposed during the first trimester included children of mothers who had redeemed a prescription 30 days prior to pregnancy or in the first trimester. Former use was defined as redemption of corticosteroid prescriptions (for one systemic and/or two local corticosteroids) at any time prior to the 30 days before pregnancy.

In Denmark most local and all systemic corticosteroids are dispensed by prescription only. The Danish Registry of Medicinal Product Statistics (RMPS) maintains records on type of drug (according to the Anatomical Therapeutic Chemical (ATC) classification system) and date of prescription reimbursement for all prescribed medications dispensed from pharmacies nationwide. Information from the RMPS was also linked to our study subjects through the CRN. We did not have information on corticosteroids administered in hospital.

\section{Asthma}

Asthma in offspring was defined as an inpatient, outpatient or emergency room (ER) diagnosis of asthma and/or by redemption of at least two prescriptions for $\beta$-2-agonists and two prescriptions for inhaled corticosteroids. A similar algorithm, requiring only one prescription for a $\beta$-2-agonist and one prescription for an inhaled corticosteroid, has a positive predictive value of $80-100 \%$ in patients aged $5-45$ years. ${ }^{17}$ We obtained data on asthma diagnoses from the Danish National Registry of Patients (DNRP), coded according to WHO's International Classification of Diseases (ICD), 8th revision until the end of 1993 and 10th revision thereafter. The DNRP contains information on all inpatient discharges from nonpsychiatric acute care hospitals since 1977. Both ER and outpatient specialist clinic contacts were added in $1995 .{ }^{15}$

\section{Data on covariates}

We included a number of covariates identified as risk factors for asthma. From the DMBR we obtained information on maternal age at delivery, ${ }^{18}$ maternal smoking status, ${ }^{19}$ maternal BMI (recorded from 2004 onward), ${ }^{20}$ gender, ${ }^{2}$ gestational age, ${ }^{21}$ Apgar score,${ }^{22}$ birth order, ${ }^{23}$ birth weight ${ }^{24}$ and caesarean section. ${ }^{25}$ From the RMPS we obtained information on maternal use of medications (paracetamol, PPI, COX inhibitors and antibiotics). ${ }^{5-8}$ The DNRP was used to identify maternal asthma, maternal IBD (subdivided into ulcerative colitis and Crohn's disease (CD)), maternal diabetes, maternal chronic obstructive pulmonary disease and other maternal autoimmune diseases. ${ }^{26}$ All relevant ICD and ATC codes are provided in online supplementary appendices 1 and 2, whereas stratification by maternal disease is provided in online supplementary appendix 3 .

\section{Statistical analysis}

Offspring were followed from date of birth until date of asthma, death, emigration or the end of follow-up on 31 December 2010, whichever came first. We computed 2-year, 5-year and 10-year risk of asthma according to corticosteroid exposure, considering death as a competing risk. Cox proportional hazards regression was used to compute crude and adjusted HRs (aHRs) with 95\% CI, comparing children exposed to corticosteroids during gestation, and children of mothers who were former corticosteroid users, with unexposed children. The analyses were adjusted for maternal age, maternal smoking status, maternal use of medication (paracetamol, PPIs, COX inhibitors and antibiotics), mode of delivery, gestational age, birth order, birth weight, gender and birth year. In an additional analysis we also adjusted for maternal BMI, for which we had data from 2004 onward. Analyses were repeated according to trimester of exposure. In a separate analysis we compared children exposed to corticosteroids postnatally with children wholly unexposed (during pregnancy and postnatally). Because Danish women are encouraged to breastfeed during the first postpartum year, ${ }^{27}$ we used 
this timeframe to define the postnatal exposure period. We did a sensitivity analysis only including children who were given an asthma diagnosis (ie, excluding the medicine algorithm) as well as an analysis in which we started follow-up at the age of 5 years (a diagnosis of asthma can only be made with certainty from age 5), ${ }^{28}$ both in order to exclude children with wheezing. Additionally, we moved the start of the exposure period from 30 days prior to pregnancy to 0 and 60 days, respectively, to evaluate if this was a sensible cut-off point.

\section{Within-mother-between-pregnancy analysis}

We conducted a 'within-mother-between-pregnancy' analysis, based on the assumption that siblings share genetic and environmental factors during their upbringing. ${ }^{29}$ We identified families in which at least one sibling was exposed to corticosteroids anytime during gestation and at least one sibling was not. The unexposed siblings served as the reference cohort. Conditional logistic regression, adjusted for calendar period of birth and thereby addressing length of follow-up, was chosen over stratified Cox regression for this analysis. Logistic regression handled sibling pairs where both experienced the outcome more appropriately, all though results did not differ substantially from the stratified Cox regression. ORs with $95 \%$ CIs can be interpreted as estimates of relative risk. Model 1 was adjusted for birth period (1996-2000, 2001-2005 or 2006-2009), to account for differences in length of follow-up. Model 2, in addition, was adjusted for the same variables as in the main analysis including the general population comparison cohort. We also conducted a second 'within-parentsbetween-pregnancy' analysis restricted to full siblings, with the same parents.

Analyses were performed using SAS (V.9.2; SAS Inc, Cary, North Carolina, USA). The study was approved by the Danish Data Protection Agency (record no. 2013-41-1790).

Table 1 Characteristics of mothers of live-born children from 1 January 1996 to 31 December 2009 in Denmark according to corticosteroid drug use during pregnancy $(\mathrm{N}=877778)$

\begin{tabular}{|c|c|c|}
\hline Characteristics & $\begin{array}{l}\text { Corticosteroid use } \\
\text { during pregnancy, n (\%) }\end{array}$ & $\begin{array}{l}\text { No corticosteroid use } \\
\text { during pregnancy, } \mathrm{n}(\%)\end{array}$ \\
\hline All & $31759(100)$ & $846019(100)$ \\
\hline \multicolumn{3}{|l|}{ Age at giving birth (years) } \\
\hline$<25$ & $2363(7.4)$ & $119386(14.1)$ \\
\hline $25-29$ & $9306(29.3)$ & 295693 (35.0) \\
\hline $30-34$ & $12819(40.4)$ & 295891 (35.0) \\
\hline 35-39 & $6170(19.4)$ & 116104 (13.7) \\
\hline$\geq 40$ & $1101(3.5)$ & $18945(2.2)$ \\
\hline \multicolumn{3}{|l|}{ Use of other drugs during pregnancy } \\
\hline Antibiotics & $14817(46.7)$ & 297906 (35.2) \\
\hline Paracetamol & $554(1.7)$ & $5367(0.6)$ \\
\hline PPIs & $887(2.8)$ & $10238(1.2)$ \\
\hline NSAIDs/Coxibs & $2384(7.5)$ & $35412(4.2)$ \\
\hline \multicolumn{3}{|l|}{ Smoking during pregnancy } \\
\hline No & $25391(80.0)$ & 653391 (77.2) \\
\hline $1-10$ cigarettes/day & 3908 (12.3) & 120435 (14.2) \\
\hline 11-20 cigarettes/day & $1150(3.6)$ & 35206 (4.2) \\
\hline$>20$ cigarettes/day & $173(0.5)$ & $4962(0.6)$ \\
\hline Missing & $1137(3.6)$ & 32025 (3.8) \\
\hline \multicolumn{3}{|l|}{ Chronic disease } \\
\hline Inflammatory bowel disease (IBD) & 1037 (3.3) & $5097(0.6)$ \\
\hline Crohn's disease (CD) & $251(0.8)$ & $1775(0.2)$ \\
\hline Ulcerative colitis (UC) & $655(2.1)$ & $2793(0.3)$ \\
\hline Both & $131(0.4)$ & $529(0.1)$ \\
\hline Chronic obstructive pulmonary disease (COPD) & $178(0.6)$ & $2159(0.3)$ \\
\hline Maternal diabetes mellitus (type 1) & $116(0.4)$ & $2865(0.3)$ \\
\hline Maternal diabetes mellitus (type 2) & $120(0.4)$ & $2355(0.3)$ \\
\hline Asthma & $4431(14.0)$ & $38662(4.6)$ \\
\hline \multicolumn{3}{|l|}{ Maternal body mass index (BMI) * } \\
\hline Low & $553(1.7)$ & $16854(2.0)$ \\
\hline Normal & $8846(27.9)$ & 204289 (24.1) \\
\hline Overweight & $3131(9.9)$ & $68411(8.1)$ \\
\hline Obese and severe obesity & $1716(5.4)$ & $38215(4.5)$ \\
\hline
\end{tabular}




\section{RESULTS}

\section{Descriptive data}

We identified 877778 children born alive in Denmark from 1 January 1996 until 31 December 2009. Overall 31759 children $(3.6 \%)$ were prenatally exposed to corticosteroids and 5325 of their mothers $(0.6 \%)$ used systemic corticosteroids (of these, 3800 redeemed one prescription and 1525 redeemed two or more prescriptions). Local corticosteroids were used by 26434 mothers $(3.0 \%)$ during pregnancy. Mothers who used corticosteroids during pregnancy were older than non-users, and used other drugs more frequently during pregnancy (table 1). Children exposed to corticosteroids during gestation were more frequently delivered by caesarean section than unexposed children (table 2).

\section{Asthma in offspring}

Median follow-up time was 5.8 years for exposed and 7 years for unexposed children. The absolute risk of asthma among exposed children was 9.5\%, 15.1\% and $18.4 \%$ after 2, 5 and 10 years of follow-up, respectively. Corresponding estimates among unexposed children were $6.8 \%, 10.9 \%$ and $13.5 \%$.

Prenatal exposure to systemic corticosteroids was associated with asthma ( $\mathrm{aHR}=1.54$ (95\% CI 1.45 to 1.65$)$ ). This estimate was highest among children of mothers who redeemed only one prescription for corticosteroids, compared to children of mothers who redeemed two or more. The aHR was 1.32 (95\% CI 1.30 to 1.34) for children of former users of corticosteroids. Maternal corticosteroid use during the breastfeeding period was also associated with increased risk of asthma in offspring compared with never use ( $\mathrm{aHR}=1.19$ (95\% CI 1.15 to 1.23)).

Results were almost identical for children whose mothers used local corticosteroids during pregnancy compared with systemic use (table 3) and risk of asthma did not vary substantially according to trimester of exposure (results not shown). Estimates also did not

Table 2 Characteristics of 877778 children born in Denmark between 1 January 1996 and 31 December 2009 according to prenatal exposure to corticosteroids

\begin{tabular}{|c|c|c|}
\hline Characteristics & $\begin{array}{l}\text { Exposed to corticosteroids } \\
\text { during pregnancy, } \mathrm{n}(\%)\end{array}$ & $\begin{array}{l}\text { Not exposed to corticosteroids } \\
\text { during pregnancy, } n(\%)\end{array}$ \\
\hline \multicolumn{3}{|l|}{ Exposure period } \\
\hline Corticosteroid use 30 days prior to conception & $2246(7.1)$ & \\
\hline Exposure during first trimester & $7297(23.0)$ & \\
\hline Exposure during second and third trimester & $22216(70.0)$ & \\
\hline \multicolumn{3}{|l|}{ Gestational age (weeks) } \\
\hline $19-29$ & $96(0.3)$ & $2918(0.3)$ \\
\hline 30-36 & $1402(4.4)$ & $37965(4.5)$ \\
\hline $37-41$ & $27686(87.2)$ & $736254(87.0)$ \\
\hline $42-48$ & $2418(7.6)$ & $63202(7.5)$ \\
\hline Missing & $157(0.5)$ & $5680(0.7)$ \\
\hline \multicolumn{3}{|l|}{ Mode of delivery } \\
\hline Caesarean section & $6717(21.2)$ & $148607(17.6)$ \\
\hline Respiratory distress syndrome & $1218(3.8)$ & $29910(3.5)$ \\
\hline \multicolumn{3}{|l|}{ Apgar score } \\
\hline$<7$ & $206(0.7)$ & $5828(0,7)$ \\
\hline $7-9$ & $2136(6.7)$ & $55853(6.6)$ \\
\hline 10 & $29114(91.7)$ & 774315 (91.5) \\
\hline Missing & $303(1.0)$ & $10023(1.2)$ \\
\hline \multicolumn{3}{|l|}{ Birth weight (g) } \\
\hline 1500-2000 & $207(0.7)$ & $6003(0.7)$ \\
\hline 2000-2499 & $687(2.2)$ & $18565(2.2)$ \\
\hline 2500-2999 & $2956(9.3)$ & $86449(10.2)$ \\
\hline $3000-5000$ & 27567 (86.8) & 723099 (85.5) \\
\hline Missing & $342(1.1)$ & $11903(1.4)$ \\
\hline \multicolumn{3}{|l|}{ Gender } \\
\hline Girl & $15236(48.0)$ & 411944 (48.7) \\
\hline Boy & $16523(52.0)$ & 434075 (51.3) \\
\hline \multicolumn{3}{|l|}{ Birth order } \\
\hline 1 & 12020 (37.9) & 365101 (43.2) \\
\hline 2 & $12688(40.0)$ & 314768 (37.2) \\
\hline$\geq 3$ & 7051 (22.2) & $166150(19.6)$ \\
\hline \multicolumn{3}{|l|}{ Birth year } \\
\hline 1966-2000 & $9881(31.1)$ & 312128 (36.9) \\
\hline 2001-2005 & 11524 (35.4) & 297823 (35.2) \\
\hline 2006-2009 & 10624 (33.5) & 236068 (27.9) \\
\hline
\end{tabular}


Table 3 Crude and adjusted HRs for asthma in children born in Denmark between 1996 and 2009, according to prenatal exposure to local or systemic corticosteroids at any time during gestation.

\begin{tabular}{lrll}
\hline & $\mathbf{N}$ & Crude HR (95\% Cl) & Adjusted HR (95\% Cl) \\
\hline No corticosteroid use & & 1.00 (ref) & 1.00 (ref) \\
Systemic treatment & 5325 & $1.73(1.62$ to 1.85$)$ & $1.54(1.45$ to 1.65$)$ \\
$\quad 1$ redeemed prescription & 3800 & $1.73(1.61$ to 1.87$)$ & $1.60(1.48$ to 1.72$)$ \\
$\geq 2$ redeemed prescriptions & 1525 & $1.73(1.53$ to 1.95$)$ & $1.42(1.26$ to 1.60$)$ \\
Local treatment & 26434 & $1.45(1.40$ to 1.50$)$ & $1.45(1.40$ to 1.50$)$ \\
Former maternal use & 162949 & $1.36(1.34$ to 1.38$)$ & $1.32(1.30$ to 1.34$)$ \\
\hline
\end{tabular}

${ }^{*}$ Adjusted for maternal age, maternal smoking, maternal use of antibiotics, paracetamol, PPIs or anti-inflammatory drugs, mode of delivery, birth year, birth weight, gestational age, birth order and gender.

change when asthma was strictly defined as a hospital diagnosis of asthma (ie, not including asthma medication), when follow-up began at age 5 , or with additional adjustment for maternal BMI (results not shown). Finally, changing the exposure period from 30 days prior to pregnancy to 0 and 60 days prior to pregnancy did not affect the estimates (data not shown).

\section{Sibling comparison}

The 'within-mother-between-pregnancy' analysis indicated a minor increase in asthma risk in siblings exposed to corticosteroids, compared to unexposed siblings $(\mathrm{aOR}=1.11$ (95\% CI 0.98 to 1.25$)$ ) (table 4). Restricting the analysis to children with the same parents, instead of just the same mother, did not change the estimates substantially, aOR=1.04 (95\% CI 0.92 to 1.18$)$.

\section{DISCUSSION}

The attenuated association in the 'within-motherbetween-pregnancy' analysis, bordering on no association, strongly indicates the presence of confounding by family related or other factors in the analyses including a general population comparison cohort. This is further underlined by the positive association of former maternal corticosteroid use with asthma in offspring, and by comparable aHRs for local and systemic use. Thus, these population-based data do not support a strong causal association between exposure to corticosteroid therapy during gestation and increased asthma risk.

To the best of our knowledge, no previous studies have investigated the association between maternal corticosteroid use at any time during pregnancy and risk of asthma in offspring. A Canadian study by Pole et $a l^{14}$ reported that corticosteroid therapy administered just prior to birth to induce fetal lung maturation was associated with increased risk of asthma in offspring, between 3 and 5 years of age (aHR 1.19 (95\% CI 1.03 to 1.39)). But the risk was decreased after 8 years of age (aHR 0.74 (95\% CI 0.54 to 1.03$)$ ). The indication of the corticosteroid therapy examined in the Canadian study, fetal lung maturation, is more closely related to asthma development than the maternal indications present in our study. This may hamper direct comparison of the results of the two studies.

Of the mothers diagnosed with asthma (43 093) only around $10 \%$ (4431) were in corticosteroid therapy during their pregnancy. This might be due to the fact that our definition of asthma does not take into account the different degrees of asthma, as mild asthma can be managed with $\beta$-2-agonists when symptoms occur. Another possible explanation might be that women could have enough medication for long periods of time, and thus does not redeem prescriptions during their pregnancy. We cannot, however, rule out the possibility of misclassification due to a wrong/hastily given diagnosis.

The strengths of our study include its large sample size, long and complete follow-up and use of populationbased medical registries. Thus, selection and recall biases were virtually eliminated. Furthermore, the availability of registry data on family relations made the 'within-mother-between-pregnancy' analysis possible.

Table 4 Crude and adjusted ORs for asthma in children prenatally exposed to corticosteroids compared with unexposed siblings.

\begin{tabular}{|c|c|c|c|}
\hline & $\mathbf{N}$ & $\begin{array}{l}\text { Model } 1 \\
\text { OR }(95 \% \mathrm{Cl})\end{array}$ & $\begin{array}{l}\text { Model } 2 \\
\text { OR }(95 \% \mathrm{Cl})\end{array}$ \\
\hline Unexposed children (at least one sibling has been prenatally exposed) & 5957 & 1.00 (ref) & 1.00 (ref) \\
\hline Exposed children (at least one sibling has been prenatally unexposed) & 4542 & $1.12(1.00$ to 1.24$)$ & $1.11(0.98$ to 1.25$)$ \\
\hline
\end{tabular}


The fact that we did not have information on over-the-counter medication (containing very low doses of corticosteroids) and in-hospital administered corticosteroids and the exclusion of topical corticosteroids is a potential source of bias. However, we have no reason to believe that this exposure was different between the exposed and the unexposed, and thus the use of these medications would have biased our results towards the null.

Potential misclassification of exposure to corticosteroids should be noted, since we only know that mothers redeemed the prescribed medication at a pharmacy. We had no data on actual drug use and dosage of the different types of drugs. However, corticosteroid treatment during pregnancy is followed quite closely by physicians, which increases compliance. Furthermore, we cannot rule out some misclassification of children with wheezing as asthma patients, as these children could have been given a diagnosis of asthma hastily or have redeemed more than two prescriptions for corticosteroids and $\beta$-2-agonists. We therefore used a validated algorithm and made it even stricter to identify asthma outcomes ${ }^{17}$; however, the validated algorithm was done for the prescription-based diagnosis. However, we cannot rule out that some children might have been misclassified if misdiagnosed. Despite this we do not believe that these potential sources of bias were able to conceal a strong causal association in the 'within-mother-betweenpregnancy' analysis. Frisell et $a l^{30}$ recently demonstrated that sibling designs are susceptible to random measurement error of exposures, leading to attenuation of association estimates. However, part of the analyses that were based on comparison with the general population comparison cohort further indicate the presence of confounding in this comparison. Thus, there was a positive association between former maternal use of corticosteroids and asthma in offspring and the aHRs for maternal use of local and systemic corticosteroids were virtually similar.

In conclusion, the aetiology of asthma likely involves common risk factors for maternal corticosteroid treatment and asthma in offspring, such as environmental or genetic factors. However, based on the 'within-motherbetween-pregnancy' analysis, we do not consider prenatal exposure to corticosteroids to be a strong risk factor for asthma development, which may apply to immunosuppression during pregnancy in general.

Contributors HTS conceived the study. All authors contributed to the design of the study, the interpretation of the results, and the writing process. $A B$ drafted the initial manuscript. TF performed the statistical analyses.

Funding This study was supported by the Danish Council for Technology and Innovation and the Clinical Epidemiology Research Foundation, Aarhus, Denmark.

Competing interests None.

Provenance and peer review Not commissioned; externally peer reviewed. Data sharing statement No additional data are available.
Open Access This is an Open Access article distributed in accordance with the Creative Commons Attribution Non Commercial (CC BY-NC 3.0) license, which permits others to distribute, remix, adapt, build upon this work noncommercially, and license their derivative works on different terms, provided the original work is properly cited and the use is non-commercial. See: http:// creativecommons.org/licenses/by-nc/3.0/

\section{REFERENCES}

1. The International Study of Asthma and Allergies in Childhood (ISAAC) steering committee. Worldwide variation in prevalence of symptoms of asthma, allergic rhinoconjunctivitis, and atopic eczema: ISAAC. Lancet 1998;351:1225-32.

2. Subbarao P, Becker A, Brook JR, et al. Epidemiology of asthma: risk factors for development. Expert Rev Clin Immunol 2009;5:77-95.

3. Blacquiére MJ, Timens W, Melgert BN, et al. Maternal smoking during pregnancy induces airway remodeling in mice offspring. Eur Respir J 2009;33:1133-40.

4. Scholtens $\mathrm{S}$, Wijga $\mathrm{AH}$, Brunekreef $\mathrm{B}$, et al. Maternal overweight before pregnancy and asthma in offspring followed for 8 years. Int $J$ Obes (Lond) 2010;34:606-13.

5. Andersen $A B$, Farkas DK, Mehnert $F$, et al. Use of prescription paracetamol during pregnancy and risk of asthma in children: a population-based Danish cohort study. Clin Epidemiol 2012;4:33-40.

6. Dehlink E, Yen E, Leichtner AM, et al. First evidence of a possible association between gastric acid suppression during pregnancy and childhood asthma: a population-based register study. Clin Exp Allergy 2009;39:246-53.

7. Burdan F, Staroslawska E, Szumilo J. Prenatal tolerability of acetaminophen and other over-the-counter non-selective cyclooxygenase inhibitors. Pharmacol Rep 2012;64:521-7.

8. Murk W, Risnes KR, Bracken MB. Prenatal or early-life exposure to antibiotics and risk of childhood asthma: a systemic review. Pediatrics 2011;127:125-38.

9. Ngoc PL, Gold DR, Tzianabos AO, et al. Cytokines, allergy and asthma. Curr Opin Allergy Clin Immunol 2005;5:161-6.

10. Elenkov IJ. Glucocorticoids and the Th1/Th2 balance. Ann N Y Acad Sci 2004:1024:138-46.

11. Tegethoff M, Greene N, Olsen J, et al. Inhaled glucocorticoids during pregnancy and offspring pediatric diseases: a national cohort study. Am J Respir Crit Care Med 2012;185:557-63.

12. Tegethoff M, Pryce $C$, Meinlschmidt G. Effects of intrauterine exposure to synthetic glucocorticoids on fetal, newborn, and infant hypothalamic-pituitary-adrenal axis function in humans: a systematic review. Endocr Rev 2009;30:753-89.

13. Mortimer KJ, Tattersfield AE. Benefit versus risk for oral, inhaled, and nasal glucocorticosteroids. Immunol Allergy Clin North Am 2005;25:523-39.

14. Pole JD, Mustard CA, To T, et al. Antenatal steroid therapy for fetal lung maturation and the subsequent risk of childhood asthma: a longitudinal analysis. J Pregnancy 2010;2010:789748.

15. Sørensen HT, Christensen T, Schlosser HK, et al. Use of medical databases in clinical epidemiology. 2nd edn. SUN-TRYK: Aarhus University, 2009.

16. Blenstrup LT, Knudsen LB. Danish registers on aspects of reproduction. Scand J Public Health 2011;39(7 Suppl):79-82.

17. Osborne ML, Vollmer WM, Johnson RE, et al. Use of an automated prescription database to identify individuals with asthma. J Clin Epidemiol 1995;48:1393-7.

18. Laerum BN, Svanes C, Wentzel-Larsen T, et al. Young maternal age at delivery is associated with asthma in adult offspring. Respir Med 2007;101:1431-8.

19. Burke H, Leonardi-Bee J, Hashim A, et al. Prenatal and passive smoke exposure and incidence of asthma and wheeze: systematic review and meta-analysis. Pediatrics 2012;129:735-44.

20. Harpsoe MC, Basit S, Bager $P$, et al. Maternal obesity, gestational weight gain, and risk of asthma and atopic disease in offspring: a study within the Danish National Birth Cohort. J Allergy Clin Immunol 2013;131:1033-40.

21. Tedner SG, Örtqvist AK, Almqvist C. Fetal growth and risk of childhood asthma and allergic disease. Clin Exp Allergy 2012;42:1430-47.

22. Xu B, Pekkanen J, Järvellin MR. Obstetric complications and asthma in childhood. J Asthma 2000;37:589-94.

23. Goldberg S, Israeli E, Schwartz S, et al. Asthma prevalence, family size, and birth order. Chest 2007;131:1747-52.

24. Brew BK, Marks GB. CAPS (Childhood Asthma Prevention, Study) Investigators. Perinatal factors and respiratory health in children. Clin Exp Allergy 2012;42:1621-9. 
25. Kolokotroni O, Middleton N, Gavatha M, et al. Asthma and atopy in children born by caesarean section: effect modification by family history of allergies-a population based cross-sectional study. BMC Pediatr 2012:12:179.

26. Hemminki K, Li X, Sundquist $\mathrm{K}$, et al. Familial association of inflammatory bowel diseases with other autoimmune and related diseases. Am J Gastroenterol 2010;105:139-47.

27. http://www.sst.dk/publ/Publ2009/CFF/Boernesundhed/Amning09.pdf
28. Bacharier LB, Boner A, Carlsen $\mathrm{KH}$, et al. Diagnosis and treatment of asthma in childhood: a PRACTALL consensus report. Allergy 2008;63:5-34.

29. Lindström K, Lindblad F, Hjern A. Preterm birth and attention-deficit/ hyperactivity disorder in schoolchildren. Pediatrics 2011;127:858-65.

30. Frisell T, Öberg S, Kuja-Halkola R, et al. Sibling comparison designs: bias from non-shared confounders and measurement error. Epidemiology 2012;23:713-20. 